\title{
Characterization of a virulent Leptospira interrogans strain isolated from an abandoned swimming pool
}

\author{
Karine M. Forster ${ }^{1}$, Daiane D. Hartwig ${ }^{1}$, Fabiana K. Seixas ${ }^{1}$, Alan J.A. McBride ${ }^{1,2}$, \\ Leonardo G. Monte ${ }^{1}$, Ana Lúcia C. Recuero ${ }^{3}$, Claudiomar S. Brod ${ }^{3}$, \\ Cláudia P. Hartleben ${ }^{1}$, Marta Amaral ${ }^{1}$, Odir A. Dellagostin ${ }^{1}$ \\ ${ }^{1}$ Núcleo de Biotecnologia, Centro de Desenvolvimento Tecnológico, Universidade Federal de Pelotas, \\ Pelotas, RS, Brazil. \\ ${ }^{2}$ Instituto Gonçalo Moniz, Fundação Oswaldo Cruz, Ministério da Saúde, Salvador, BA, Brazil. \\ ${ }^{3}$ Centro de Controle de Zoonoses, Universidade Federal de Pelotas, Pelotas, RS, Brazil.
}

Submitted: February 22, 2012; Approved: July 2, 2012.

\begin{abstract}
Pathogenic Leptospira spp. are the etiological agents of leptospirosis, an important disease of both humans and animals. In urban settings, $L$. interrogans serovars are the predominant cause of disease in humans. The purpose of this study was to characterize a novel Leptospira isolate recovered from an abandoned swimming pool. Molecular characterization through sequencing of the rpoB gene revealed $100 \%$ identity with $L$. interrogans and variable-number tandem-repeat (VNTR) analysis resulted in a banding pattern identical to L. interrogans serogroup Icterohaemorrhagiae, serovar Copenhageni or Icterohaemorrhagiae. The virulence of the strain was determined in a hamster model of lethal leptospirosis. The lethal dose $50 \%\left(\mathrm{LD}_{50}\right)$ was calculated to be two leptospires in female hamsters and a histopathological examination of infected animals found typical lesions associated with severe leptospirosis, including renal epithelium degeneration, hepatic karyomegaly, liver-plate disarray and lymphocyte infiltration. This highly virulent strain is now available for use in further studies, especially evaluation of vaccine candidates.
\end{abstract}

Key words: Leptospira, Leptospirosis, Virulent, VNTR, rроB.

\section{Introduction}

Leptospirosis is a disease caused by pathogenic spirochetes belonging to the Leptospira genus (Faine et al., 1999). It has a worldwide distribution and affects both humans and other animals (Bharti et al., 2003), with more than 500,000 cases a year (WHO, 1999), however, it is still a neglected disease (WHO, 2011). The pathogen is maintained in the environment by susceptible or reservoir hosts that shed the bacteria in their urine, contaminating soil and waterways (Levett, 2001). Humans, an accidental host, typically become infected by direct or indirect contact with the urine of infected animals, particularly during seasonal flooding associated with heavy rainfall (Ko et al., 1999, Lau et al., 2010). Although human leptospirosis is considered an occupational hazard in developed countries, it is now more common in urban slums or in subsistence farming communities in underdeveloped countries (Mcbride et al., 2005, Ko et al., 2009). A wide variety of animals serve as potential reservoir hosts. The relative importance of a given reservoir species depends on the area, population density, the type of housing and the occupational and leisure activities of the local residents (Reis et al., 2008). Leptospires are considerably resilient and are the only spirochaetes that can survive outside the host (Faine et al., 1999). Isolation and characterization of pathogenic leptospires is important for the development of new vaccines and for improving diagnosis.

This study reports the isolation of a virulent Leptospira strain from a sample of water collected from a swimming pool in Southern Brazil and its characterization 
by sequencing of the rроB gene and variable-number tandem-repeat (VNTR) analysis.

\section{Materials and Methods}

\section{Leptospira spp. isolation and culture conditions}

A sample of water was collected from an abandoned swimming pool, which contained dead possums and rats, in the city of Pelotas, RS, Brazil. A few drops of this water were used to inoculate $5 \mathrm{~mL}$ Ellinghausen-McCulloughJohnson-Harris (EMJH) liquid medium supplemented with Leptospira Enrichment EMJH (Difco, BD Diagnostics, Sparks, MD, USA) and the cultures were incubated at $30{ }^{\circ} \mathrm{C}$. After seven days, the culture was centrifuged at $5,000 \mathrm{x} g$ for $5 \mathrm{~min}$, the supernatant was passed through a $0.22 \mu \mathrm{m}$ filter (Millipore, Billerica, MA, USA) and the filtrate was used to inoculate another tube of EMJH liquid medium. After nine in vitro passages, uncontaminated spirochete cells could be observed by darkfield microscopy. The isolate was named Spool, and stored in liquid nitrogen.

\section{Genomic DNA extraction}

A $10 \mathrm{~mL}$ culture grown for 7 days in EMJH medium was inactivated in a water bath at $56^{\circ} \mathrm{C}$ for $30 \mathrm{~min}$, centrifuged at 13,000 $\mathrm{x} g$ for $5 \mathrm{~min}$, and DNA was extracted using Illustra Bacterium GenomicPrep Mini Spin kit following the manufacturer's instructions (GE Healthcare, São Paulo, SP, Brazil). The extracted DNA was analyzed by agarose gel electrophoresis to evaluate its integrity and quality, and stored at $-20^{\circ} \mathrm{C}$.

\section{Partial sequencing of the $r p o B$ gene}

The hyper-variable region between base pairs 1900 and 2500 of the $r p o B$ gene was amplified with primers Lept 1900f (5'-CCTCATGGGTTCCAACATGCA) and Lept 2500r (5'-CGCATCCTCRAAGTTGTAWCCTT) as previously described (La Scola et al., 2006). DNA sequencing was performed using a MegaBACE 500 DNA sequencer and Dynamic ET-terminator technology (GE Healthcare). The resulting sequence was submitted to basic local alignment search tool (BLAST) alignment (www.ncbi.nlm.nih.gov/BLAST) to identify the Leptospira spp. by alignment with $r p o B$ sequences in GenBank.

\section{VNTR analysis}

Seven discriminatory primers (VNTR4, VNTR7, VNTR9, VNTR10, VNTR11, VNTR19 e VNTR23) were used to characterize the isolate as previously described (Majed et al., 2005). The size of the amplified products was analyzed by agarose gel electrophoresis as described previously (Sambrook and Russell, 2000). Genomic DNA purified from $L$. interrogans serovar Copenhageni strain Fiocruz L1-130 was used as a positive control.

\section{Western blotting}

For Western blotting, a whole-cell extract was separated by $12 \%$ sodium dodecyl sulphate-polyacrylamide gel electrophoresis (SDS-PAGE), and transferred to a nitrocellulose membrane Hybond ECL (GE Healthcare) as previously described (Sambrook and Russell, 2000). After blocking the membranes were incubated with the anti-LipL32 1D9 MAb at 1:500 dilution in PBS or antiLigA and LigB polyclonal mono-specific mouse sera at 1:100 dilution in PBS. After three washes with PBS containing $0.05 \%(\mathrm{v} / \mathrm{v})$ Tween $20($ PBS- $\mathrm{T})$, the membranes were incubated with an anti-mouse $\operatorname{IgG}$ peroxidase conjugate diluted in PBS-T. The reaction was developed with 4-chloro-1-naphthol (Sigma) after five washes with PBST. The BenchMark Pre-Stained Protein Ladder (Invitrogen, São Paulo, SP, Brazil) was used as molecular weight marker.

\section{Virulence testing and histopathology}

The virulence of the isolate was confirmed using the hamster model of lethal leptospirosis. The animals were housed at the animal facility of the Federal University of Pelotas (UFPel) and maintained in accordance with the guidelines of the Ethics Committee in Animal Experimentation, UFPel throughout the study period. Leptospires were counted in a Petroff-Hauser counting chamber (Fisher Scientific, Pittsburgh, PA, USA) as previously described (Faine et al., 1999). Two nine-week-old male hamsters were inoculated intraperitoneally with $10^{8}$ leptospires. Clinical symptoms were monitored by daily observations during the study period and moribund animals were euthanized immediately. Post-mortem, the kidneys, lungs and liver were collected and stored in $10 \%$ formalin for histopathological examination after staining with haematoxylin and eosin. One of the kidneys from each animal was macerated and inoculated into EMJH liquid medium for re-isolation.

\section{Determination of the $\mathrm{LD}_{50}$}

Forty golden Syrian hamsters (nine-week old, 20 males and 20 females) were infected intraperitoneally with 10 fold serial dilutions ( $10^{4}$ to $10^{0}$ leptospires) in a final volume of $1 \mathrm{~mL}$ PBS (see Table 1). Animals were monitored daily for clinical symptoms until 30 days post-infection, where upon all survivors were euthanized. The $\mathrm{LD}_{50}$ was calculated by the method as previously described (Reed and Muench, 1938).

\section{Results}

The presence of dead rats and a possum in a swimming pool that had not received any type of water treatment for more than one year prompted us to try to isolate leptospires from a sample of the water. Although the primary culture was contaminated with other bacteria, a few spirochetes could be observed under darkfield microscopy. 
After passing the culture through a $0.22 \mu \mathrm{m}$ filter and sub-culturing in liquid EMJH medium for nine passages, a pure culture was obtained.

After DNA extraction, PCR amplification and partial sequencing of the $r p o B$, BLAST alignment with sequences in GenBank reported $100 \%$ identity with the $r p o B$ gene from $L$. interrogans (data not shown). To further characterize the isolate, VNTR analysis was performed using seven VNTR loci. Analysis of the electrophoresis profile of the amplified VNTR fragments revealed an identical pattern between the isolate and the L. interrogans serovar Copenhageni L1-130 strain (Figure 1).
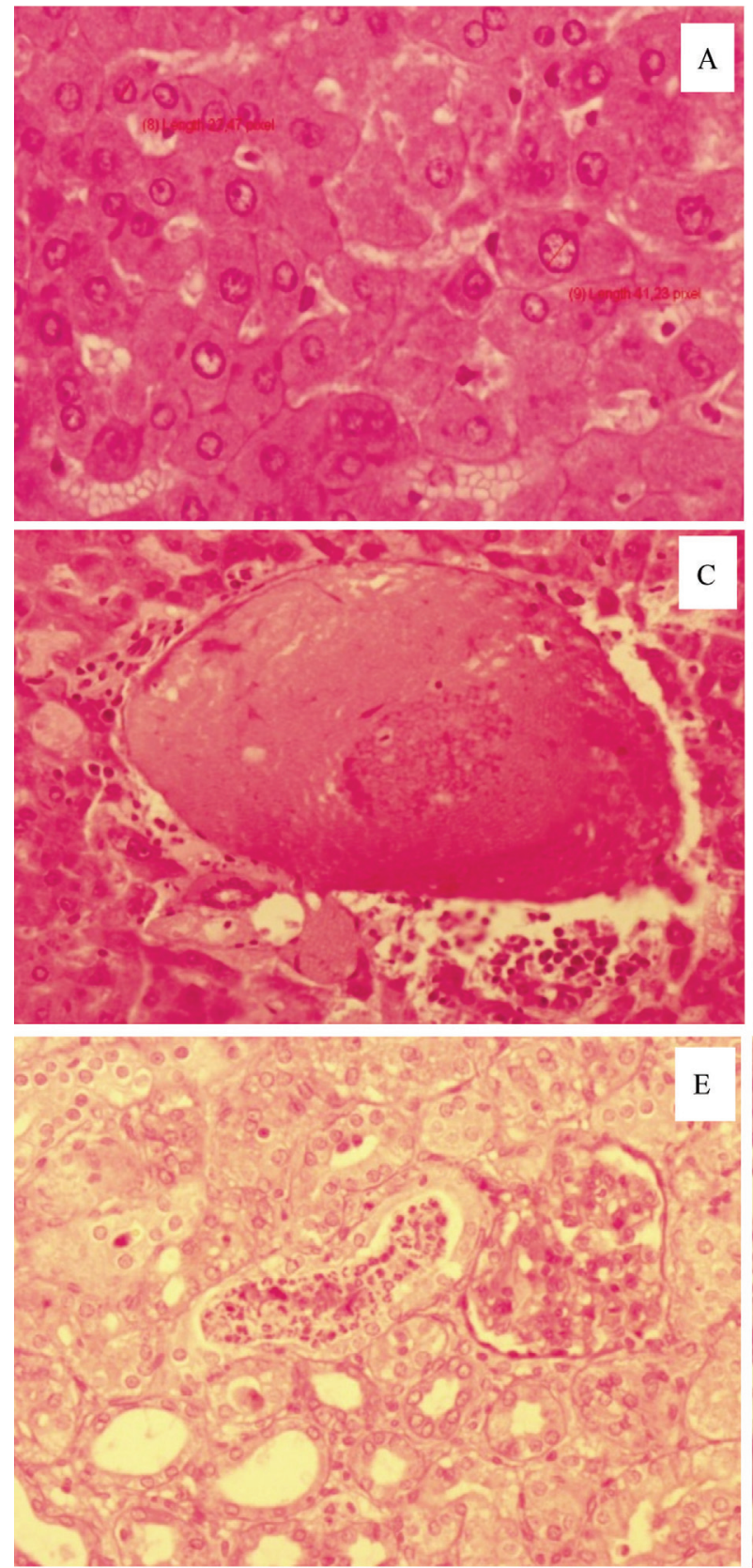

To further confirm the pathogenic status of the isolate, expression of the LipL32, LigA and LigB proteins, which are unique to pathogenic Leptospira spp., was evaluated by Western blotting. Expression of all three of the antigens was observed (Figure 2), confirming that the isolate was a pathogenic Leptospira strain.

In a confirmatory test for the virulence of the isolate, all hamsters that were inoculated with $10^{8}$ leptospires went on to develop lethal leptospirosis (data not shown). Typically, clinical symptoms and death were observed on the second to fourth day post-infection (pi). The symptoms included: dehydration, ruffled hair coat, decreased activity
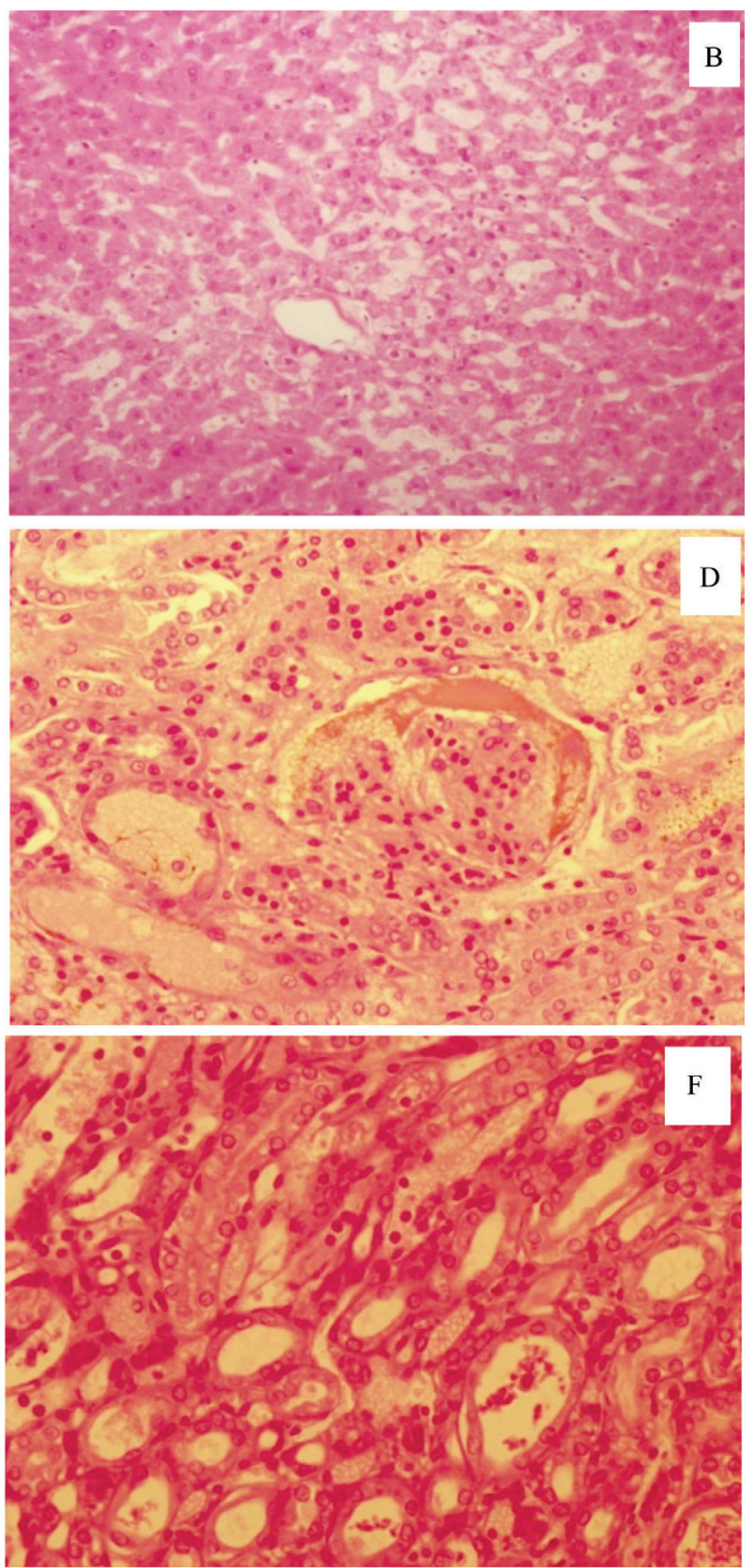

Figure 1 - Electrophoresis in $0.8 \%$ agarose gel. Columns 1 and 2 with molecular marker (1 kb DNA ladder, Invitrogen); (a) L. interrogans L1-130 used as positive control; (b) L. interrogans strain SPool. 


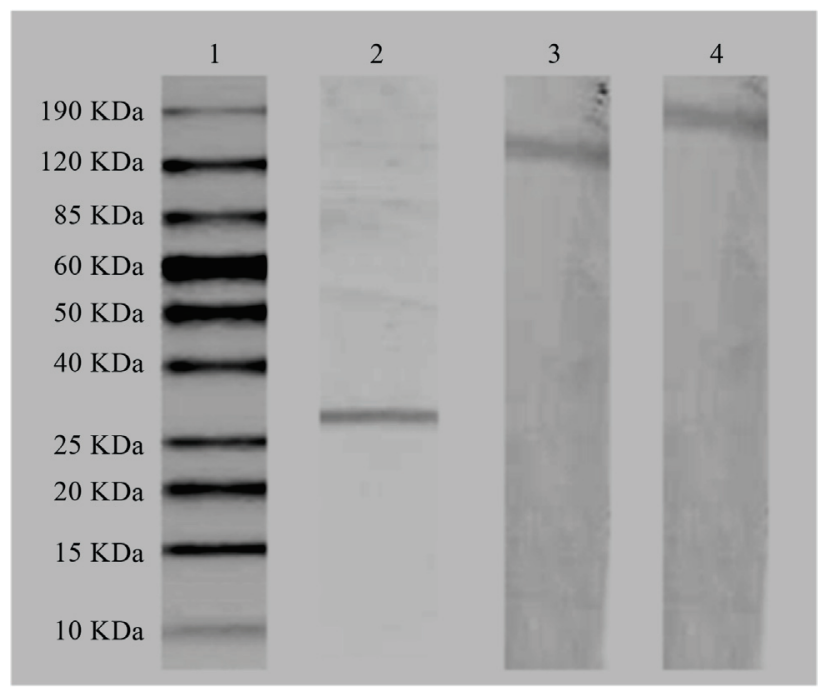

Figure 2 - Western blot of SPool isolate cell extract probed with different antibodies. Lane 1, BenchMark Pre-Stained ladder; lane 2, anti-LipL32; lane 3, anti-LigA; lane 4, anti-LigB.
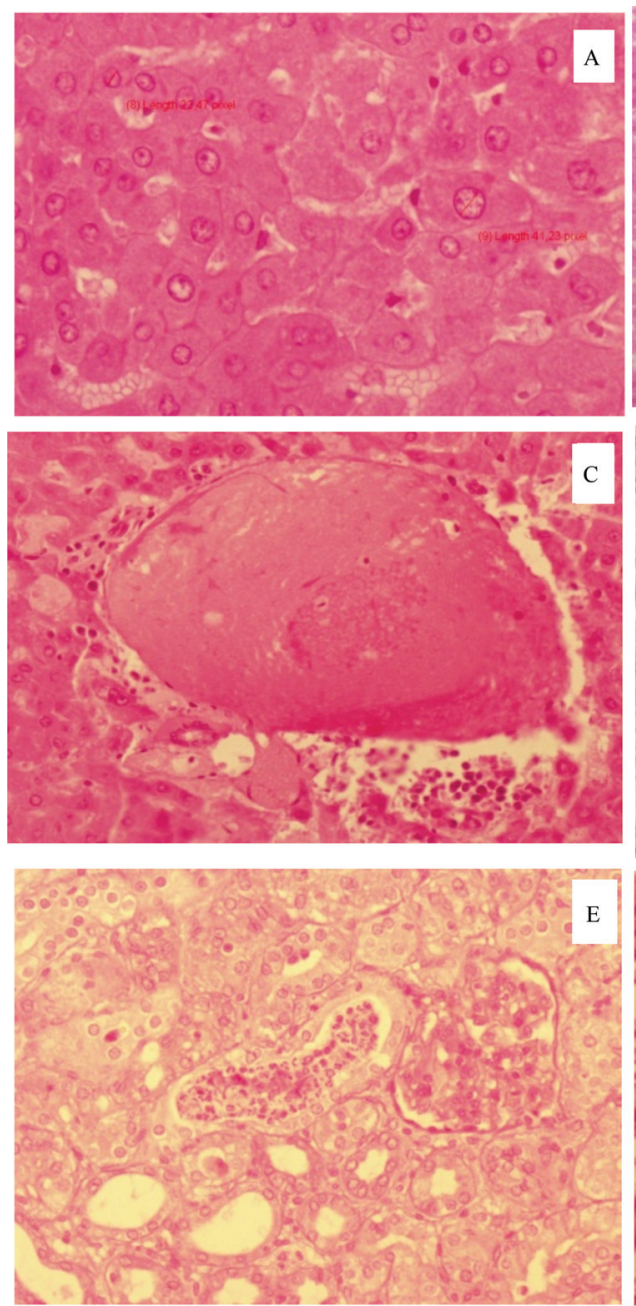

and isolation. In addition, we observed severe external haemorrhaging, swelling and complete closure of the eyes. The histopathological results showed that the infected animals developed renal epithelium degeneration and hepatic cells with karyomegaly, diffuse loss of cohesion (liverplate disarray), and lymphocyte infiltration (Figure 3).

In the experiments to determine the $\mathrm{LD}_{50}$, death was observed to begin on day $11 \mathrm{pi}$, and continued to day $22 \mathrm{pi}$ (Figure 4). None of the hamsters inoculated with 10 or more leptospires survived the infection. Inoculation with one leptospire resulted in death for $25 \%(1 / 4)$ of female and $50 \%(2 / 4)$ of male hamsters. The $\mathrm{LD}_{50}$ was calculated by the regression method of Reed and Muench, and was $\sim 2$ and $\sim 1$ leptospires for female and male hamsters, respectively.

\section{Results and Discussion}

The isolation and characterization of region-specific Leptospira isolates is essential towards understanding the
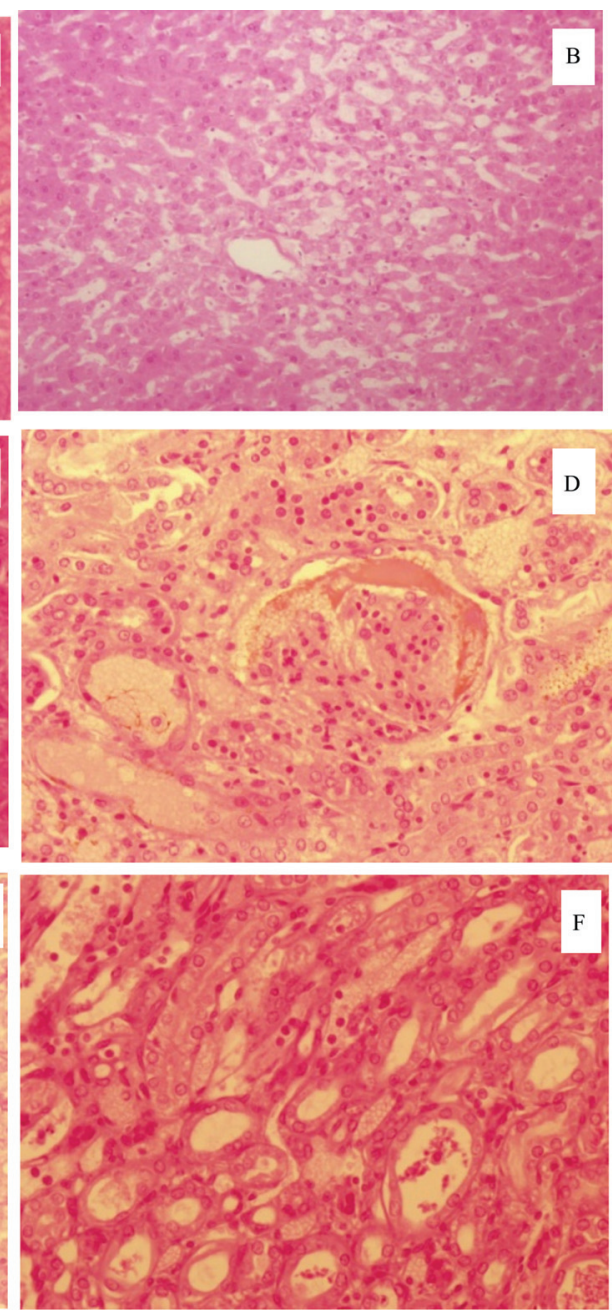

Figure 3 - Typical lesions of leptospirosis in a 9-week-old hamster that died four days after infection with L. interrogans strain SPool. (A) Hepatic cells with karyomegaly. (B) Diffuse loss of cohesion (liver-plate disarray) of liver cells. (C) Lymphocyte infiltration around the central vein. (D) Degenerate renal glomerulum with haemorrhagic oedema. (E) Leukocyte infiltration in the renal tissue. (F) Renal tissue with macrophages cells. 


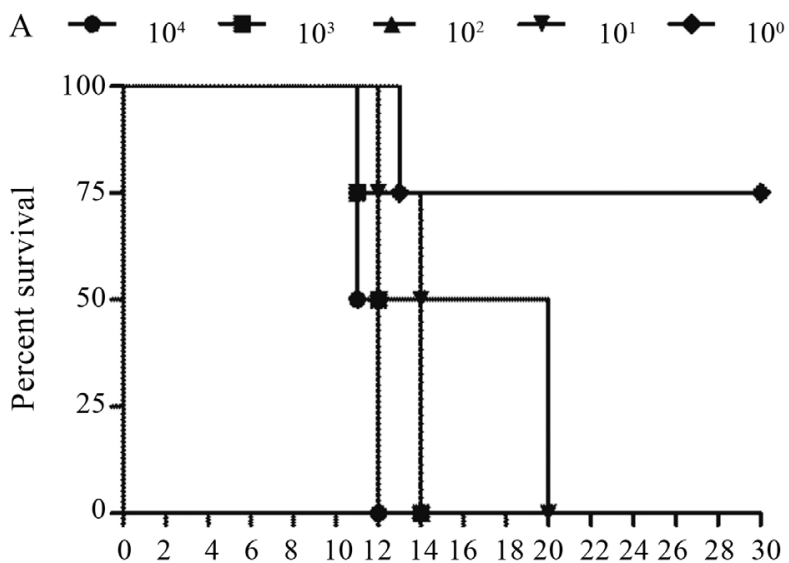

B

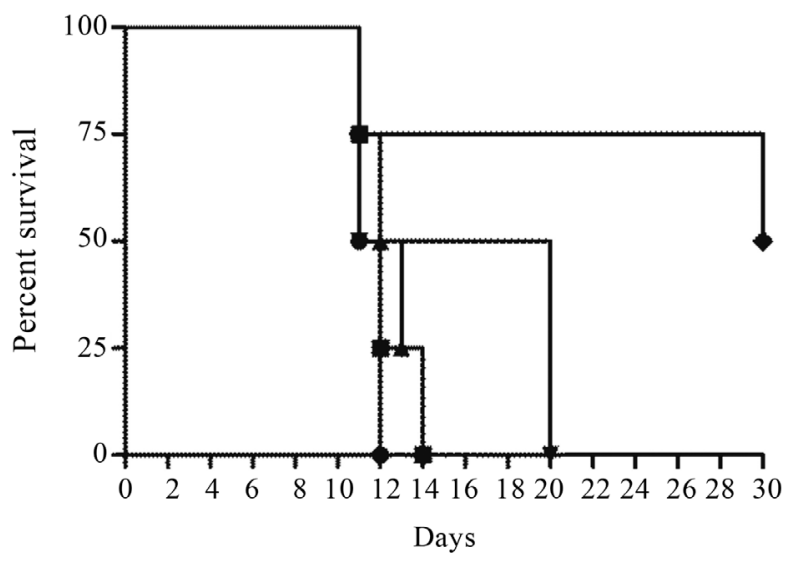

Figure 4 - Survival curves for calculation of the $\mathrm{LD}_{50}$ for the $L$. interrogans SPool isolate. Groups of female (A) and male (B) hamsters $(\mathrm{n}=4)$ infected with $10^{4}, 10^{3}, 10^{2}, 10^{1}$ and $10^{0}$ leptospires.

epidemiology and improving diagnosis of leptospirosis (Bourhy et al., 2010). In this study, we obtained an isolate of Leptospira from a sample of water collected from a swimming pool in the city of Pelotas, in the Southeast of Brazil. This swimming pool had not been cleaned or treated for more than one year and two rat and one possum carcasses were observed. After nine passages in vitro and filtering to remove contaminants, a pure culture was obtained, as determined by darkfield microscopy.

Molecular characterization by partial sequencing of the rpoB gene and VNTR analysis have become the standard molecular techniques used for identification to the species and serogroup level of Leptospira spp. (La Scola et al., 2006, Cerqueira and Picardeau, 2009). VNTR analysis was carried out using the loci described by Majed et al. (2005). Based on the rрoB sequence alignments with sequences in GenBank, the isolate was identified as $L$. interrogans, belonging to the Icterohaemorrhagiae serogroup. The serovar was determined to be either Icterohaemorrhagiae or Copenhageni, however, it was not possi- ble to discriminate between the two as these serovars have identical VNTR profiles (Figure 1).

The virulence of Leptospira spp. is known to be strain dependent (Faine et al., 1999) and this was highlighted by the sequencing of two L. borgpetersenii serovar Hardjo strains, only one of which was virulent in hamsters (Bulach et al., 2006). The hamster model reproduces the pathology observed in acute forms of human leptospirosis, as previously described (Haake, 2006, Dellagostin et al., 2011). The typical pathology includes acute cell swelling and multifocal regeneration tubular foci and interstitial nephritis. Using the hamster model of leptospirosis the virulence of the SPool isolate was confirmed and the $\mathrm{LD}_{50}$ was calculated to be approximately two and one leptospires in the female and male models, respectively. Furthermore, various strains of Leptospira spp. can cause host-dependent symptoms and some of the symptoms are characteristic of a particular strain. In this study, the symptoms caused by the SPool strain included swelling of the eyelids resulting in complete closing of the eyes. This was an unusual finding and has not previously been reported.

To further characterize the SPool isolate the expression of several pathogenic-specific antigens was evaluated by indirect immunofluorescence and Western blotting. The expression of LipL32, the immunodominant surfaceexposed antigen was confirmed using an anti-LipL32 MAb (Fernandes et al., 2007). The lig genes encode virulence determinants involved in host-pathogen interactions and they have been used for diagnosis and as vaccine candidates (Dellagostin et al., 2011). LigA and LigB expression was detected using specific polyclonal mouse sera.

Due to the severe side effects associated with the currently used whole-cell (bacterin) type leptospirosis vaccines there is a need for an alternative, recombinant vaccine for the prevention of leptospirosis (Dellagostin et al., 2011). A more complete evaluation of the capacity of vaccine candidates to induce a cross-protective immune response requires a well characterized panel of virulent strains representing the Leptospira serovars of public health and veterinary importance (Silva et al., 2008). With the isolation of the SPool strain we report on the availability of an additional strain for evaluation of vaccine candidates. Previous studies of vaccine candidates reported variable efficacies and this was suggested to be due to virulence of the challenge strain (Adler and Moctezuma, 2010). Weakly virulent strains requiring challenge doses of $\geq 10^{6}$ leptospires often resulted in survivors in the control groups. The $\mathrm{LD}_{50}$ of the SPool strain is approximately 2 leptospires, one of the lowest described to date.

In conclusion, we report the isolation and characterization of a pathogenic isolate of $L$. interrogans obtained from a swimming pool. This highly virulent strain will be used in experiments evaluating the protection afforded by novel recombinant vaccine candidates against leptospirosis currently being evaluated in our laboratories. 


\section{References}

Adler B, de la Pena MA (2010) Leptospira and leptospirosis. Vet Microbiol 140:287-296.

Bharti AR, Nally JE, Ricaldi JN, Matthias MA, Diaz MM, Lovett MA, Levett PN, Gilman RH, Willig MR, Gotuzzo E, Vinetz JM (2003) Leptospirosis: a zoonotic disease of global importance. Lancet Infect Dis 3:757-771.

Bourhy P, Collet L, Clement S, Huerre M, Ave P, Giry C, Pettinelli F, Picardeau M (2010) Isolation and characterization of new Leptospira genotypes from patients in Mayotte (Indian Ocean). PLoS Negl Trop Dis 4:e724.

Bulach DM, Zuerner RL, Wilson P, Seemann T, McGrath A, Cullen PA, Davis J, Johnson M, Kuczek E, Alt DP, Peterson-Burch B, Coppel RL, Rood JI, Davies JK, Adler B (2006) Genome reduction in Leptospira borgpetersenii reflects limited transmission potential. Proc Natl Acad Sci USA 103:14560-14565.

Cerqueira GM, Picardeau M (2009) A century of Leptospira strain typing. Infect Genet Evol 9:760-768.

Dellagostin OA, Grassmann AA, Hartwig DD, Felix SR, da Silva EF, McBride AJ (2011) Recombinant vaccines against leptospirosis. Hum Vaccine 7: 1215-1224.

Faine S, Adler B, Bolin C, Perolat P (1999) Leptospira and Leptospirosis 2nd ed. MediSci, Melbourne.

Fernandes CP, Seixas FK, Coutinho ML, Vasconcellos FA, Seyffert N, Croda J, McBride AJ, Ko AI, Dellagostin OA, Aleixo JA (2007) Monoclonal antibodies against LipL32, the major outer membrane protein of pathogenic Leptospira: production, characterization, and testing in diagnostic applications. Hybridoma (Larchmt) 26:35-41.

Haake DA (2006) Hamster model of leptospirosis. Curr Protoc Microbiol Chapter 12, Unit, 12E.12-13.

Ko AI, Galvao RM, Ribeiro Dourado CM, Johnson WDJr, Riley LW (1999) Urban epidemic of severe leptospirosis in Brazil. Salvador Leptospirosis Study Group. Lancet 354:820-825.
Ko AI, Goarant C, Picardeau M (2009) Leptospira: the dawn of the molecular genetics era for an emerging zoonotic pathogen. Nat Rev Microbiol 7:736-747.

La SB, Bui LT, Baranton G, Khamis A, Raoult D (2006) Partial rpoB gene sequencing for identification of Leptospira species. FEMS Microbiol Lett 263:142-147.

Lau CL, Smythe LD, Craig SB, Weinstein P (2010) Climate change, flooding, urbanisation and leptospirosis: fuelling the fire? Trans R Soc Trop Med Hyg 104:631-638.

Levett PN (2001) Leptospirosis. Clin Microbiol Rev 14:296-326.

Majed Z, Bellenger E, Postic D, Pourcel C, Baranton G, Picardeau $M$ (2005) Identification of variable-number tandem-repeat loci in Leptospira interrogans sensu stricto. J Clin Microbiol 43:539-545.

McBride AJ, Athanazio DA, Reis MG, Ko AI (2005) Leptospirosis. Curr Opin Infect Dis 18:376-386.

Reed LJ, Muench H (1938) A simple method of estimating fifty percent endpoints. Am J Hyg 27:493-497.

Reis RB, Ribeiro GS, Felzemburgh RD, Santana FS, Mohr S, Melendez AX, Queiroz A, Santos AC, Ravines RR, Tassinari WS, Carvalho MS, Reis MG, Ko AI (2008) Impact of environment and social gradient on Leptospira infection in urban slums. PLoS Negl Trop Dis 2:e228.

Sambrook J, Russell DW (2013) Molecular Cloning: A Laboratory Manual 3rd ed. CSHL Press, Cold Spring Harbor.

Silva EF, Santos CS, Athanazio DA, Seyffert N, Seixas FK, Cerqueira GM, Fagundes MQ, Brod CS, Reis MG, Dellagostin OA, Ko AI (2008) Characterization of virulence of Leptospira isolates in a hamster model. Vaccine 26:38923896.

WHO (1999) Leptospirosis worldwide, 1999. Wkly Epidemiol Rec 74:237-242.

WHO (2011) Leptospirosis: an emerging public health problem. Wkly Epidemiol Rec 86:45-50.

All the content of the journal, except where otherwise noted, is licensed under a Creative Commons License CC BY-NC. 